\title{
The Water Polymorphism and the Liquid-Liquid Transition from Transport Data
}

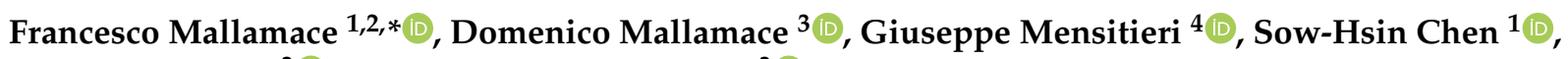 \\ Paola Lanzafame ${ }^{3}\left[\right.$ and Georgia Papanikolaou ${ }^{3}$ (i)
}

1 Department of Nuclear Science and Engineering, Massachusetts Institute of Technology, Cambridge, MA 02139, USA; sowhsin@mit.edu

2 CNR ISC, UOS Roma Sapienza, Physics Department, Sapienza University of Rome, 00185 Roma, Italy

3 Departments of ChiBioFarAm Section of Industrial Chemistry, University of Messina, CASPE-INSTM, V.le F. Stagno d'Alcontres 31, 98166 Messina, Italy; mallamaced@unime.it (D.M.); paola.lanzafame@unime.it (P.L.); georgia.papanikolaou@unime.it (G.P.)

4 Department of Chemical, Materials and Industrial Production Engineering, University of Naples Federico II, P.le Tecchio 80, 80125 Napoli, Italy; mensitie@unina.it

* Correspondence: mallamac@mit.edu; Tel.: +39-340-233-5213

check for

updates

Citation: Mallamace, F.; Mallamace,

D.; Mensitieri, G.; Chen, S.-H.;

Lanzafame, P.; Papanikolaou, G. The

Water Polymorphism and the

Liquid-Liquid Transition from

Transport Data. Physchem 2021, 1 ,

202-214. https://doi.org/10.3390/

physchem1020014

Academic Editors: Vincenzo Barone and Tullio Scopigno

Received: 31 May 2021

Accepted: 17 August 2021

Published: 25 August 2021

Publisher's Note: MDPI stays neutral with regard to jurisdictional claims in published maps and institutional affiliations.

Copyright: (c) 2021 by the authors. Licensee MDPI, Basel, Switzerland. This article is an open access article distributed under the terms and conditions of the Creative Commons Attribution (CC BY) license (https:// creativecommons.org/licenses/by/ $4.0 /)$.
Abstract: NMR spectroscopic literature data are used, in a wide temperature-pressure range (180-350 K and $0.1-400 \mathrm{MPa}$ ), to study the water polymorphism and the validity of the liquid-liquid transition (LLT) hypothesis. We have considered the self-diffusion coefficient $D_{S}$ and the reorientational correlation time $\tau_{\theta}$ (obtained from spin-lattice $T_{1}$ relaxation times), measured, respectively, in bulk and emulsion liquid water from the stable to well inside the metastable supercooled region. As an effect of the hydrogen bond (HB) networking, the isobars of both these transport functions evolve with $T$ by changing by several orders of magnitude, whereas their pressure dependence become more and more pronounced at lower temperatures. Both these transport functions were then studied according to the Adam-Gibbs model, typical of glass forming liquids, obtaining the water configurational entropy and the corresponding specific heat contribution. The comparison of the evaluated $C_{P, \text { conf }}$ isobars with the experimentally measured water specific heat reveals the full consistency of this analysis. In particular, the observed $C_{P, \text { conf }}$ maxima and its diverging behaviors clearly reveals the presence of the LLT and with a reasonable approximation the liquid-liquid critical point (LLCP) locus in the phase diagram.

Keywords: water; local order; relaxation times; self-diffusion; polymorphism

\section{Introduction}

Water, starting from the perspective of biology, has a basic role in many research fields and technological applications, regardless of whether it is in bulk or confined [1]. In chemical physics, it is of importance due to its unusual thermodynamics, compared to normal liquids, and as a prototype of supercooled liquids [2]. This is reflected in its well-known anomalies for almost all of its properties as a function of thermodynamic variables, especially below its melting temperature $T_{m}$ down to the homogeneous nucleation temperature $\left(T_{h}\right)$. Examples are represented by the pressure $(P)$ and temperature $(T)$ behaviors of its density $(\rho)$ and the thermodynamic response functions (isobaric specific heat $\left(C_{P}\right)$, the compressibility (isothermal $\kappa_{T}$ and adiabatic $\kappa_{S}$ ), and the expansivity $\left(\alpha_{P}\right)$ ) all related to the volume $(\delta V)$ or entropy $(\delta S)$ local fluctuations. For common and regular liquids, these fluctuations are positively correlated and decrease as $T$ decreases; for water, below $T_{m}$, they not only grow but become anticorrelated so that an $V$ increase brings an entropy decrease due to a growing local order accompanied by the observed diverging (critical like) behaviors in the mentioned response functions [3].

Another relevant characteristic of water which reasonably seems to be the basis of its anomalies is the polymorphism that characterizes it in all its phases including the liquid 
one polymorphism [4-7]. Although the polymorphism of the solid water crystalline phase has been known for a long time, i.e., the ice has many different structural forms ranging from the ice Ic to ice XII [8], that of amorphous water is a relatively recent discovery [9-11]. So, from the certainty of this "polyamorphism", the idea of a liquid polymorphism [12-16] was proposed.

Specifically, the amorphous water phases have different densities: the water highdensity amorphous phase (HDA) $[9,11]$ and the low-density amorphous phase (LDA) have been known since 1935 [17], and finally the VHDA (very high-density amorphous phase) [18]. Of relevant interest is that these two amorphous phases can be transformed into each other, respectively, through a reversible first order transition [10,19]. Furthermore, at ambient pressure, the LDA, if heated, undergoes a glass to liquid transition (at about $130 \mathrm{~K}$ ) into a highly viscous fluid and then crystallizes at $T_{x}=150 \mathrm{~K}$. On this basis, the liquidliquid transition hypothesis (LLT) based on the liquid polymorphism was developed [4]. A model, related to an MD study, is nowadays central in water studies, being at the base of the liquid-liquid critical hypothesis (LLCP or second critical point in distinction to the vapor-liquid one). Like in the glass, liquid water has two liquid forms of different densities (the high- and low-density liquids, respectively, HDL and LDL); they coexist and, depending on $P$ and $T$, can change one into the other by means of a first order transition: the liquid-liquid transition hypothesis (LLT). Whereas the HDL has a disordered structure (made of monomers, dimers and trimers), the LDL is characterized by an "open" structure governed by a networking process with a tetrahedral symmetry due to the noncovalent attractive hydrogen bonding (HB) interaction [4].

For precision, together with the $\mathrm{HB}$, the water molecules also interact by the Coulomb repulsion between electron lone pairs on adjacent oxygen atoms and two $\mathrm{H}-\mathrm{O}$ covalent bonds originating from the sharing of the electron lone pairs. The first one dominates in the stable and supercooled regimes and the repulsive potentials mainly influence the water physics from above the boiling temperature $\left(T_{b}\right)$ in the sub-critical and critical regions.

In particular, the LDL tetrahedral symmetry is that of ordinary ice, with four nearest neighbors around the water molecule (also acting as a H-donor to two of them and a $\mathrm{H}$-acceptor for the other two). In ice, this network is permanent while the liquid water tetrahedrality is, instead, local and transient. It should be noted that a pressure increase contrasts these ordering effects, whereas a $T$ decrease both involves its growth in size and stability; experiments show that the HB lifetime strongly increases (many orders of magnitude) from picoseconds values characteristic of the stable liquid water [20].

The tetrahedral LDL local networking, as well as the liquid polymorphism, originates the entropy decrease and the diverging behavior observed in water functions, explaining the observed water anomalies and complexity. In addition, the cited studies have allowed a more precise definition of the water phase diagram reported in Figure 1. The LLCP is estimated to be located near $200 \mathrm{~K}$ and at a pressure of $\sim 200 \mathrm{MPa}$, very far from the locus of the vapor-liquid critical point $\mathrm{CP}\left(T_{C}=647.1 \mathrm{~K}, P_{C}=22.064 \mathrm{MPa}\right)$. However, the bulk liquid water, in principle, cannot exist stably in the region between the homogeneous nucleation temperature $\left(T_{h}\right)$ and that in which the ultra-viscous liquid obtained from the fusion of LDA crystallizes $\left(T_{x}\right)$, and the LLCP seems to be located just inside this region, called "no man's land" [2]. Although such a region $\left(T_{h}-T_{x}\right)$ is open to MD simulation studies, some expedients have been used to gain experimental insight. Examples are confined water in nanopores (smaller than the nucleation centers) [21], around or inside macromolecules, in solutions, in ice, in emulsion [22-24] and micellar systems [25] or by melting a multimolecular thickness of an ice surface [26]. In particular, in this last case and for water in nanopores water can be easily maintained in the liquid state also in all the range $T_{h}-T_{x}$ and the LDA can be also achieved [21,26,27]. 


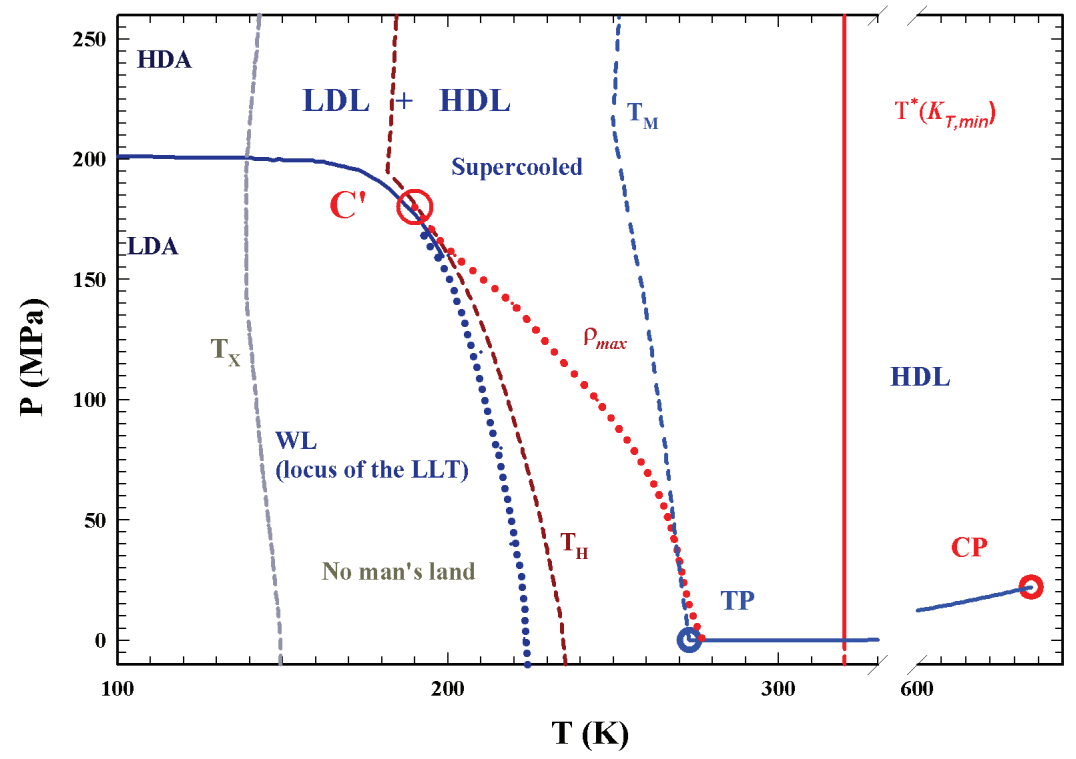

Figure 1. The $P-T$ water phase diagram. All the lines characterizing the chemical physics of the system in the liquid and disordered phases (glasses) are illustrated together with the homogeneous nucleation temperature $\left(T_{h}\right)$ and that of crystallization of the ultra-viscous liquid $\left(T_{x}\right)$ defining the "no man's land". The Widom line (characteristic of liquid-liquid transition) is also proposed together with those of the maximum density $\left(\rho_{\max }\right)$ and the melting temperature $\left(T_{m}\right)$, the critical point $C_{P}$ and the estimated (by MD) LLCP $\left(C^{\prime}\right)[2] . T^{*}$ is the temperature of the minimum of the isothermal compressibility $\kappa_{T}$ [28]. Finally, the regions of the polymorphism (LDL + HDL) and those for $T>T^{*}$ composed only of HDL are shown.

In this way, many important water properties due to the polymorphism and related to the LLT (and LLCP) were discovered. Very relevant in the present context are the results obtained from MD studies [29], pointing to the existence of the so called Widom line (WL) which identifies the $P-T$ locus of the maximum in the $\delta V$ and $\delta S$ fluctuations where thermodynamic response functions reach their extremes (minimum with negative values in the $\alpha_{P}$ and maxima in $C_{P}$ and $\kappa_{T}$ ). Other experimental results concerning the water dynamics and structure related with the Widom line and the LLT are at ambient pressure: (a) the dynamic crossover from a fragile to a strong glass-forming material, originally predicted by Angell [30] and observable at $T_{L} \simeq 225 \mathrm{~K}$ [31] that is also the locus of the Stokes-Einstein relation violation (due to the onset of the dynamic heterogeneities and the decoupling between the translational and rotational modes); (b) the compressibility maximum [32,33]. The $P-T$ locus of the WL was investigated by using neutron scattering [34], and the recent studies (experimental and simulation ones) on the isothermal compressibility agree with the related findings [35-39]. Another phenomenon observed by using confined water is the existence of a density minimum $[40,41]$, as predicted more than a century ago by Percy W. Bridgman [42] and subsequently confirmed by computational studies [36-38].

Many of the main suggestions regarding the water thermodynamics come from the large number of accurate computational studies [43], but the LLCP (inside the supercooled regime) is far off being experimentally proven in a definitive way. Due to its localization inside the "no man's land", it proved to be elusive in all the experimental attempts, although the water polymorphism and the LLT have been tried [44]. Nevertheless, the liquid polymorphism, which is favored by the temperature decrease and the corresponding growth of the hydrophilic interaction represented by the hydrogen bond, has been widely proven [5].

Very recently, according to the Adam-Gibbs model (developed to clarify the cooperative relaxation processes in glass-forming liquids [45]) and by using dynamical data (self-diffusion at ambient pressure and in a very large temperature range $[21,26]$ ), it has 
been shown that the specific heat in the supercooled water is largely due to configurational effects, and therefore to the LLT [46,47]. In such a way, the calculated configurational specific heat $C_{P, \text { conf }}$ was compared with that measured experimentally, thus confirming the fact that the temperature of the corresponding maximum is coincident (see Figure 2). Such a maximum, as theoretically predicted in terms of polymorphism [48], also defines the WL. The present work deals with an extension of such an analysis to a wider range of the $P-T$ phase diagram including transport data for pressures up to $400 \mathrm{MPa}$ and inside the supercooled region. The aim, by essentially using bulk water literature data $[20,22,49]$, is, on the one hand, to gain more information on the water thermodynamics and, on the other, to obtain more precise signals than those currently available from the LLCP and verify its localization. It must be stressed that these frame transport function data (such as self-diffusion and relaxation) are very relevant because their values provided experimental evidence of LLT, showing, by decreasing $T$, both the Widom line and the violation of the Stokes-Einstein relation, as well as the HB networking and LDL's dominance over HDL [44].

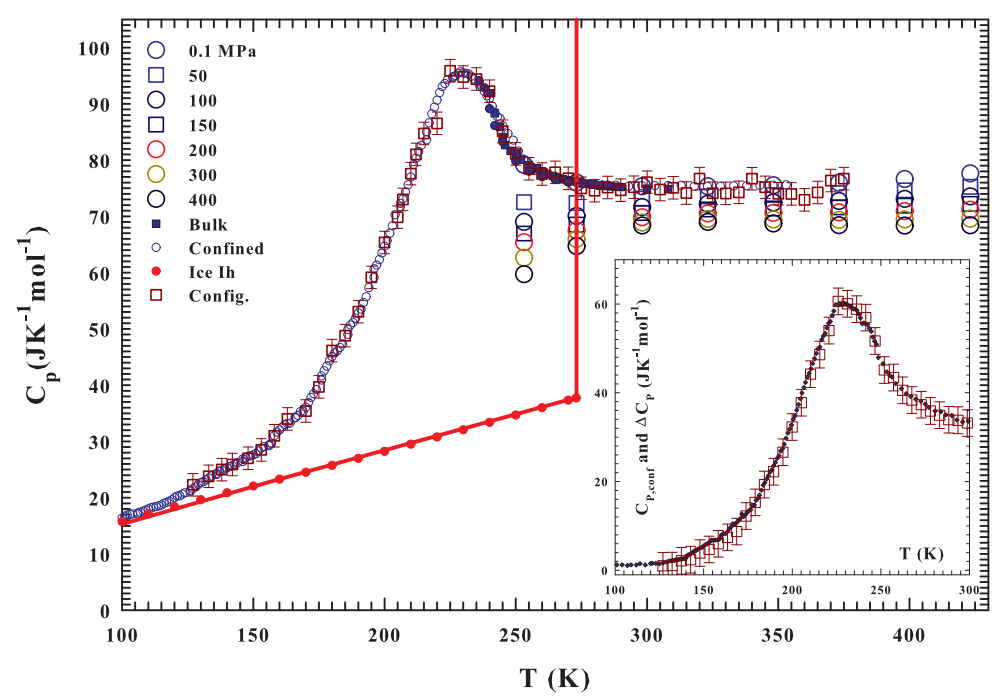

Figure 2. The figure reports the liquid water specific heat $C_{P}(T, P)$, measured in the temperature range $100-430 \mathrm{~K}$ for $0.1 \mathrm{MPa}$, and that of the Ih ice (red lines and dots [50]); for $T>250 \mathrm{~K}$ data at $P=50,100,150,200,300$ and $400 \mathrm{MPa}$ are also reported. For ambient pressure, data come from different experiments in bulk [51,52] and confined water (in nanotubes of $2.2 \mathrm{~nm}$ [47]). Data for $P>50 \mathrm{MPa}$ deal instead only with bulk water [53]. The inset shows the difference $\Delta C_{P}=C_{P, l i q}-$ $C_{P, s o l}$ (squares) and the configurational $C_{P, \text { conf }}$ evaluated by using the Adam-Gibbs model [47].

\section{Results and Discussions}

Our starting point is represented by Figure 1, which illustrates the liquid water specific heat $C_{P}(T, P)$, measured in the temperature range $100-430 \mathrm{~K}$ at $0.1 \mathrm{MPa}$, and that of the $\mathrm{Ih}$ ice (red lines and dots [50]); for $T>250, \mathrm{~K}$ data at $P=50,100,150,200,300$ and $400 \mathrm{MPa}$ are also reported. For ambient pressure data coming from different experiments in bulk [51,52] and confined water (in nanotubes of $2.2 \mathrm{~nm}$ [47]). Data for $P>50 \mathrm{MPa}$ deal instead only with bulk water [53]. We can assume [46] that, for water, the difference between the liquid and solid specific heat represents a good estimation of the configurational contribution, so that $C_{P, \text { conf }} \simeq \Delta C_{P}=C_{P, \text { liq }}-C_{P, \text { sol }}$. Therefore, in the figure inset, $\Delta C_{P}$ is reported as the difference between the specific heat values measured in the liquid and in the ice $\mathrm{Ih}$, respectively, and the $C_{P, \text { conf }}$ evaluated by using the Adam-Gibbs model (AG) theory as described in the following [47]. For the confined water, the metastable supercooled region is explored up to about $90 \mathrm{~K}$, maintaining it in the liquid state; in fact, the large peaks of latent heat that accompany solidification are absent $[47,54,55]$. 
The AG model was proposed to explain the relaxation temperature dependence in glass-forming liquids. It was detailed, in molecular-kinetic terms, by accounting the thermal effects in the size of the cooperatively rearranging regions of different energetic configurations; sizes determined by configuration restrictions, and thus expressed in terms of their configurational entropies. According to the theory, these cooperative regions have a transition probability $W(T)=F \exp \left(-z \Delta \mu / k_{B} T\right)$ that can be accounted for in terms of its size $z$ and $\Delta \mu$ (the potential energy hindering cooperative rearrangements). $F$ is a frequency factor (negligibly $T$-dependent) and $k_{B}$ is the Boltzmann constant. By expressing the cooperative region "critical size" $z^{*}$ as a function of the molar configurational entropy $S_{\text {conf }}$, the transition probability can be expressed as $W(T)=A \exp \left(-C / T S_{\text {conf }}\right)$. As the system relaxation times are related to the transition probability as $\tau(T) \propto W(T)^{-1}$, the system self-diffusion can be written as:

$$
D_{S}(T)=D_{S 0} \exp \left(-A / T S_{\text {conf }}\right)
$$

$D_{S 0}$ and $A=z \Delta \mu$ can be assumed as constant (at a given concentration). The $D_{S 0}$ value can be estimated from the $D_{S}(T)$ in the high $T$ limit. In such a way, the configurational entropy can be obtained from the system measured diffusion data (or the transport functions) and the configurational $C_{P, \text { conf }}$ was evaluated as $C_{P, \text { conf }}=T\left(\partial S_{\text {conf }} / \partial T\right)_{P}$. By using such an approach, the water configurational $C_{P, \text { conf }}$ was evaluated at ambient pressure from the bulk water diffusion data (measured and simulated in the range 373-237 K) [46,47], obtaining $D_{S 0}=1.07 \times 10^{-7} \mathrm{~m}^{2} \mathrm{~s}^{-1}$ and $A=31.75 \mathrm{kJmol}^{-1}$. The corresponding analysis was also performed by considering confined water data $[21,26,27](373-120 \mathrm{~K})$ and determining the same values of $D_{S 0}$ and $A$. The results, in terms of $C_{P, \text { conf }}$, by using this procedure are reported in Figure 2 [47].

The diffusion data used in the present analysis were obtained in 1988 from the H.-D. Lüdemann team [20] with an NMR experiment by using the pulsed field gradient spin echo technique [56] in bulk water at different pressures up to $400 \mathrm{MPa}$ and temperatures down to $200 \mathrm{~K}$. The corresponding data are illustrated in an Arrhenius plot at the different isobars in Figure 3a, together with data measured at ambient pressure in bulk and confined water used to evaluate the $C_{P, \text { conf }}$ proposed in Figure 2. As can be seen, the data show a marked difference with respect to those at ambient pressure only in the supercooled regime, where the corresponding values also increase with pressure, while for higher temperatures of the liquid stable phase the corresponding variations are significantly less pronounced. However, they show a pronounced non-Arrhenius temperature dependence and an apparently diverging correlation length of the supercooled water fluctuations. This behavior was accounted for by using the dynamic scaling behavior typical of modecoupling theory, $D_{S}(T)=D_{0}\left(T-T_{L}\right)^{\gamma}[3,57]$.

Some years before these observations, the same team proposed another NMR study on water relaxation in a meso-sized emulsion [22], hence decreasing by more than a decade the studied temperature with respect to that of the bulk water and detailing the analysis in the pressure range 1-250 MPa in steps of $25 \mathrm{MPa}$. The longitudinal proton relaxation times $T_{1}$ of the water protons have been determined, by means of the inversion recovery pulse sequence, at $100.1 \mathrm{MHz}$, at temperatures up to $186 \mathrm{~K}$, for pressures higher than $200 \mathrm{MPa}$. The corresponding proton relaxation rate $\left(R_{1}=1 / T_{1}\right)$ reported in Figure 4 at the different isobars shows a non-Arrhenius increasing dependence. It may also be noted that above the melting temperature, all the measured data, such as the $D_{S}(T)$ values in the same $T$-range, are, within the error, pressure independent. In addition, the temperature $T \simeq 315 \mathrm{~K}$ identifies, by decreasing $T$, a crossover from an Arrhenius to a non-Arrhenius behavior. From the observed behavior of the thermodynamic functions, this temperature is remarkable for water, being the locus of the onset of the HB tetrahedral structure [58,59]: it is in fact the place of the minimum, at all pressures, of isothermal compressibility $\left(\kappa_{T}(P, T)\right)$ and also represents the point where all the lines of the expansivity $\left(\alpha_{P}(P, T)\right)$ cross each other [28]. 


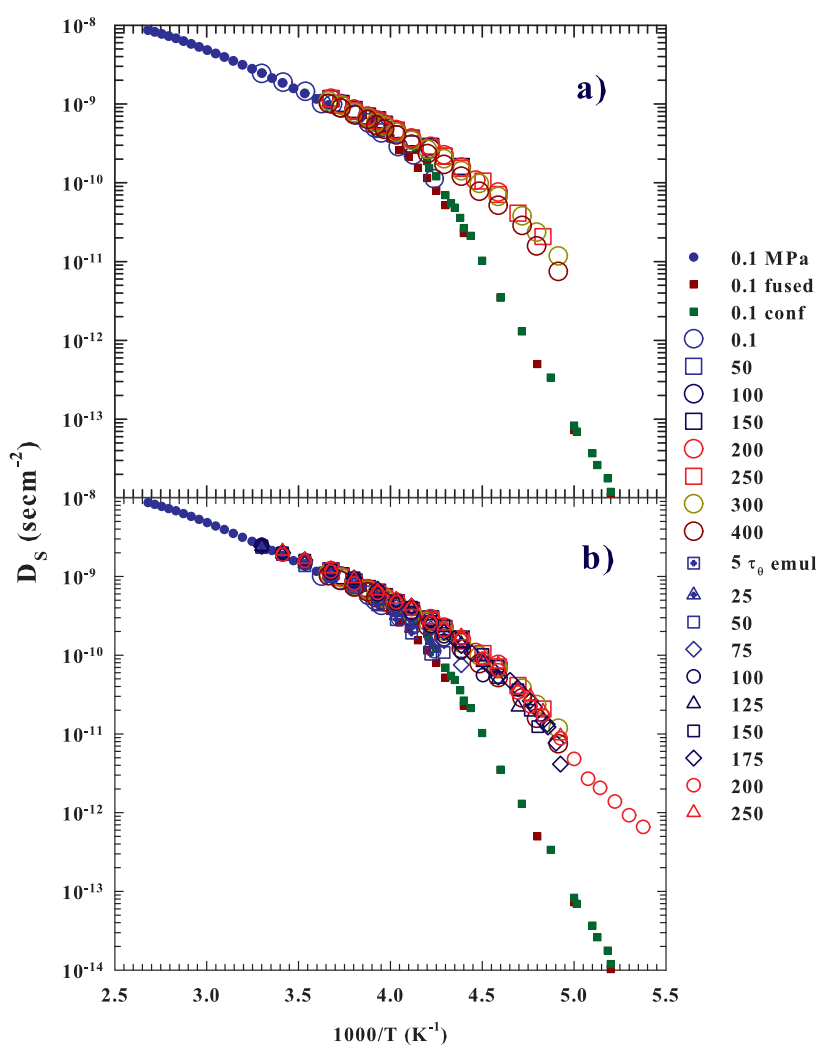

Figure 3. (a). The isobars of the bulk water self-diffusion data, measured by an NMR experiment, in the pressure range 0.1-400 MPa, are illustrated in an Arrhenius plot [20]. (b) The (a) data integrated with those corresponding to the reorientational relaxation times $\tau_{\theta}$ evaluated in terms of NMR theories from the spin-lattice relaxation times $T_{1}$ and measured in the emulsion's water (range 0.1-250 MPa) [22].

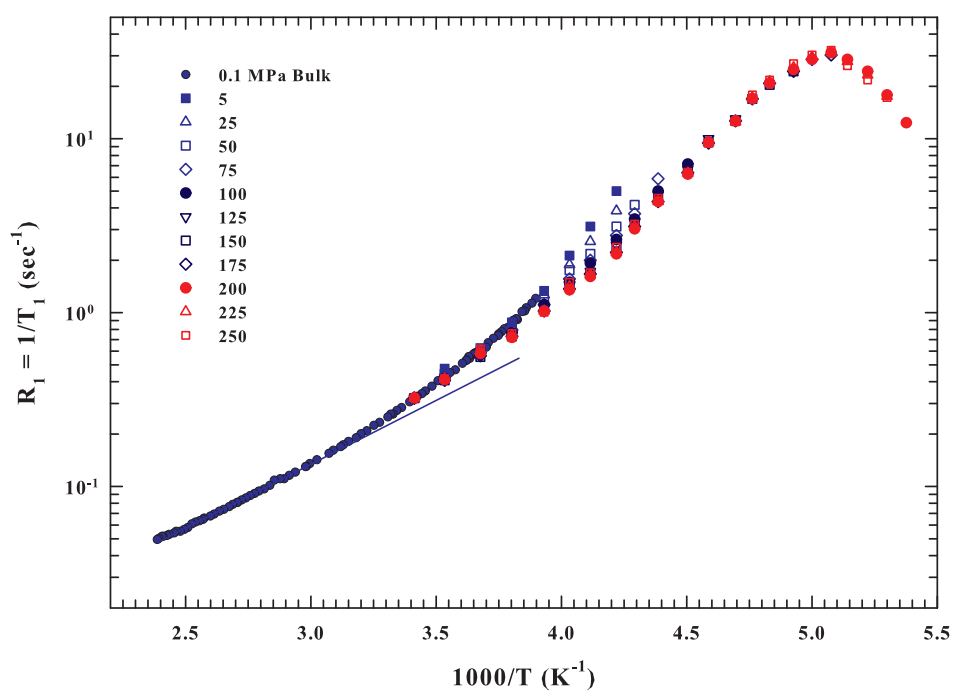

Figure 4. The different water proton relaxation rate $\left(R_{1}=1 / T_{1}\right)$ isobars, measured in the pressure range $0.1-250 \mathrm{MPa}$ [22], are proposed in an Arrhenius plot.

From $R_{1}$, according to the current theoretical models [60-62], a reorientational correlation time $\left(\tau_{\theta}\right)$ can be evaluated by assuming that the proton spin system $R_{1}$ is in general mediated via magnetic dipole couplings between nuclear magnetic moments as intramolecular or intermolecular: $\left(R_{1}\right)_{\text {Meas }}=\left(R_{1}\right)_{\text {Intra }}+\left(R_{1}\right)_{\text {Inter }}$. The first one monitors 
reorientational motions only, whereas the intermolecular one is sensitive to both positional and orientational rearrangements. In particular, the $\left(R_{1}\right)_{\text {Intra }}$ is a function of both $\tau_{\theta}$ and the Larmor frequency $\omega_{L}$ as: $\left(R_{1}\right)_{\text {Intra }}=\left(3 \gamma^{4} \hbar^{2} / 10 r^{6}\right)\left(\left(\tau_{\theta} /\left(1+\omega_{L}^{2} \tau_{\theta}^{2}\right)\right)+\left(4 \tau_{\theta} /\left(1+4 \omega_{L}^{2} \tau_{\theta}^{2}\right)\right)\right)$, so that two motion regimes, a fast $\left(\omega_{L} \tau_{\theta}<1\right)$ and a slow $\left(\omega_{L} \tau_{\theta}>1\right)$, are possible. $\gamma$ is the proton gyromagnetic ratio, $\hbar$ is the Planck's and $r$ is the distance between the water molecule hydrogens.

However, the $R_{1}$ isobars at about $200 \mathrm{MPa}$ and above run through a maximum at about $197 \mathrm{~K}$ (when $\omega_{L} \tau_{\theta} \simeq 1$ ) because an increase in pressure causes an increase in the correlation time, a situation originally predicted for normal liquids [61]. As the compression increases, the molecular motion is reflected in the correlation time. Furthermore, according to the asymptotic behavior (for $\omega_{L} \tau_{\theta} \ll 1$, it is $R_{1} \sim \tau_{\theta}$, whereas for $\omega_{L} \tau_{\theta} \gg 1$ we have $\left.R_{1} \sim 1 / \tau_{\theta}\right)$, and $\tau_{\theta}$ was also evaluated for $200 \mathrm{MPa}$ in the very slow regime [22]. According to this, Hindmann et al.'s suggestion $[63,64]$ that the water relaxation time $T$-dependence, at constant pressure, is due to two contributions (exponential) was assumed to be correct. One, at low $T$, is related with the cooperativity effects of the HB clustering and the other (at high $T$ ) to the breaking of a single HB; additionally, in these studies a relationship between viscosity, diffusion and rotational relaxation has been discussed [64].

Figure 5 shows, in the interval 200-303 K, all the $\tau_{\theta}$ isobars corresponding to the $R_{1}$, of Figure 4 , evaluated according to the above expression for $\left(R_{1}\right)_{\text {Intra }}$. For each isobar, the lowest experimentally accessible temperature increases progressively with increasing pressure, from $237 \mathrm{~K}$ at $5 \mathrm{MPa}$ to $205 \mathrm{~K}$ at $175 \mathrm{MPa}$. Only the $200 \mathrm{MPa}$ isobar, evaluated according to the asymptotic behavior, is reported up to $185 \mathrm{~K}$.

When the relaxation rates become dependent on the Larmor frequency $\left(\omega_{L}\right)$, their isobars are characterized by a dispersion and its frequency dependence revealed additional properties on the molecular motions. A study conducted at different Larmor frequencies has proved the water reorientation isotropy on a nanosecond time scale and that a single reorientational mode with a strong non-Arrhenius temperature dependence appears to be adequate to reproduce the experimental $R_{1}\left(T, P, \omega_{L}\right)$ shape [49]. The situation was related to the increasing $\mathrm{HB}$ lifetime at low temperatures and to random, transient HB network fast quasi-lattice vibrations.

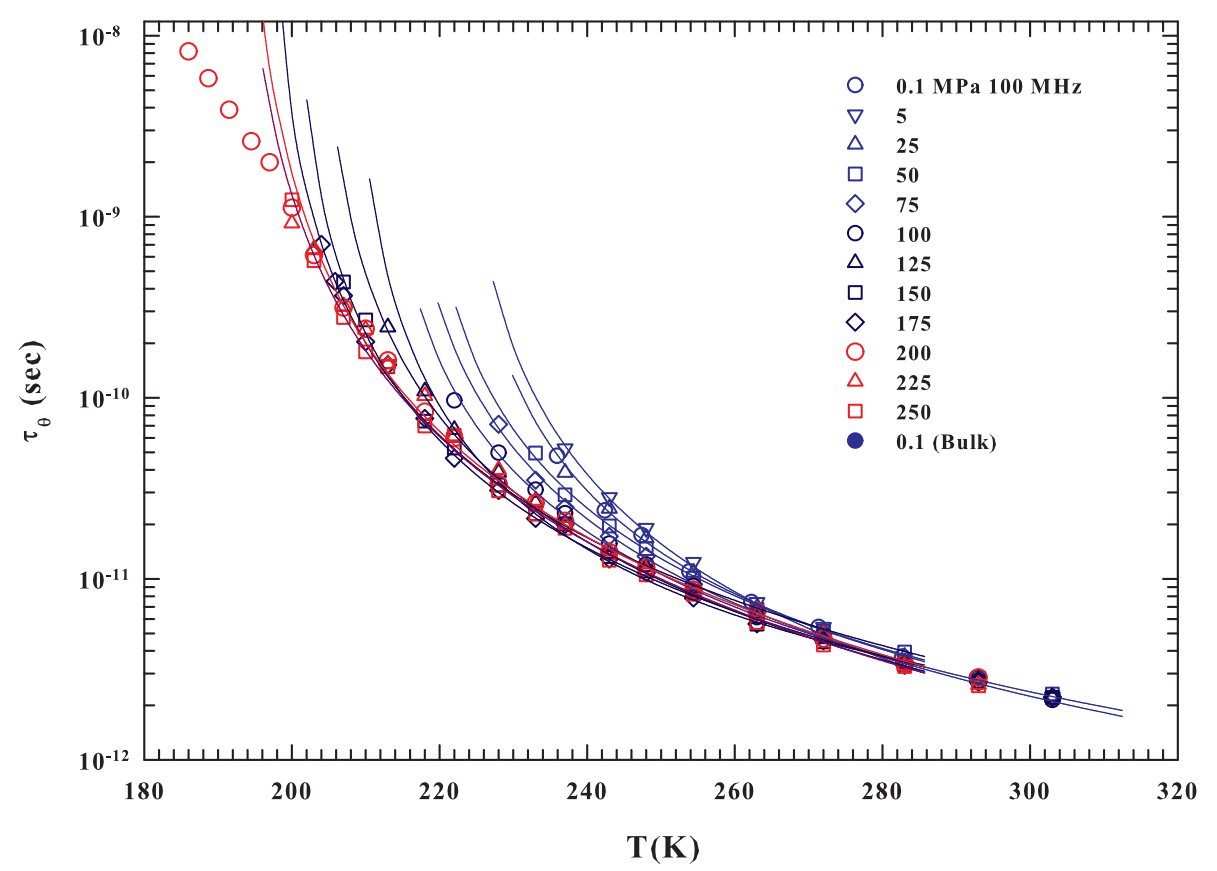

Figure 5. The reorientational correlation time $\tau_{\theta}$ isobars, corresponding to the $R_{1}$ illustrated in Figure 3, evaluated according to the current NMR theoretical models [60-62]. The dotted lines correspond to a data fitting in terms of the mode coupling theory scaling law for critical phenomena. 
According to the same analysis made on the self-diffusion data of bulk and confined supercooled water at ambient pressure, originally proposed to observe the dynamic crossover [30,31], we have fitted the NMR reorientational correlation time isobars according to the MCT reported as continuous lines in Figure 5. The obtained exponent values are for all the isobars $\gamma \simeq 2$, suggesting, for this reorientational relaxation, universal behavior, based on the energy landscape concept [65] typical of the dynamics of glass-forming liquids [66,67]. This situation seems to confirm some relationship in the deep supercooled regime between the rotational relaxation and transport parameters such as $D_{S}$. Such an analysis, even taking into account that the accessible experimental data do not reach the lowest measured in confined water, provides a reliable estimation of the crossover temperature $T_{L}$. A possible crossover at $\sim 200 \mathrm{~K}$ appears to be observable at $200 \mathrm{MPa}$.

Having this result, we tried to compare these values of $\tau_{\theta}$ with those of the diffusion shown in Figure 3a. For this, we have considered a linear relationship similar to that used in the scattering spectroscopy between longitudinal relaxation $(\tau)$ and the diffusion for low wave-vector $Q: 1 / \tau=D_{S} Q^{2}$. For the data normalization, we used the values corresponding to high temperatures which in both cases $\left(\tau_{\theta}\right.$ and $\left.D_{S}\right)$ appear to be pressure independent, obtaining the used factor of $1.587 \times 10^{20}$. Figure $3 \mathrm{~b}$ displays such a normalization showing similar behaviors between both data, thus suggesting a sort of coupling, to the same isobars, between these two transport parameters, at least for common temperatures.

The next step is the evaluation of configurational entropy and specific heats from the self-diffusion data (Figure 3) by using the AG formalism. By also considering the behavior of the data at a high temperature, we assumed for all the isobars the same value of $D_{S 0}=1.0710^{-7} \mathrm{~m}^{2} \mathrm{~s}^{-1}$ and $A=31.75 \mathrm{kJmol}^{-1}$ used for ambient pressure. Regarding the values corresponding to the rotational relaxation time, we will consider only the isobars of 175 and $200 \mathrm{MPa}$, just for a form of comparison with the effective self-diffusion data. The obtained $S_{\text {conf }}$ isobars are illustrated in Figure 6 in a linear scale for the temperature range $100-300 \mathrm{~K}$. For all reported isobars, the behavior of the data obviously reflects that observed in diffusion: continuously decreasing with decreasing $T$ and increasing with pressure (in the supercooled region) at least up to $200 \mathrm{MPa}$, a continuous and decreasing behavior can be observed with decreasing $T$, after which they seem to stabilize. The ambient pressure data, covering the entire temperature range from the stable region, well above the melting point $(300 \mathrm{~K})$, dominated by the HDL to that of the pure LDA region $(100 \mathrm{~K})$, illustrate the way in which water polymorphisms evolve with temperature. The LDA is the vitrified LDL, and LDA configurational entropy can be assumed to be essentially the continuous and slow evolution of the very low temperature $(T<160 \mathrm{~K})$ liquid component. Taking this into consideration, such a $S_{\text {conf }}$ isobar reflects, as correctly shown by N. J. Hestand and J. L. Skinner (HS) by using a logistic function for growth modeling to describe the corresponding water diffusion and radial distribution function [68], the relative amount of the two liquid components, LDL and HDL, according to a logistic function. According to this, the data flex point, where the LDL and HDL are in equivalent amounts, is to this isobar the temperature of the WL. The corresponding specific heat, reported in Figure 7, was evaluated by performing the isobars' derivative $C_{P, \text { conf }}=T\left(\partial S_{\text {conf }} / \partial T\right)_{P}$ after a fit of the entropy data by means of the same HS procedure and the same logistic function [68]. In the case of the $S_{\text {conf }}$ isobars, coming from the bulk water self-diffusion (50,100, 150, 200, 250,300 and $400 \mathrm{MPa}$ ), the data fitting was carried out with a third order polynomial, only for the rotational relaxation time $\tau_{\theta}$, evaluated from the emulsioned water relaxation rates $R_{1}, 175$ and $200 \mathrm{MPa}$, we used a polynomial of the fourth order. 


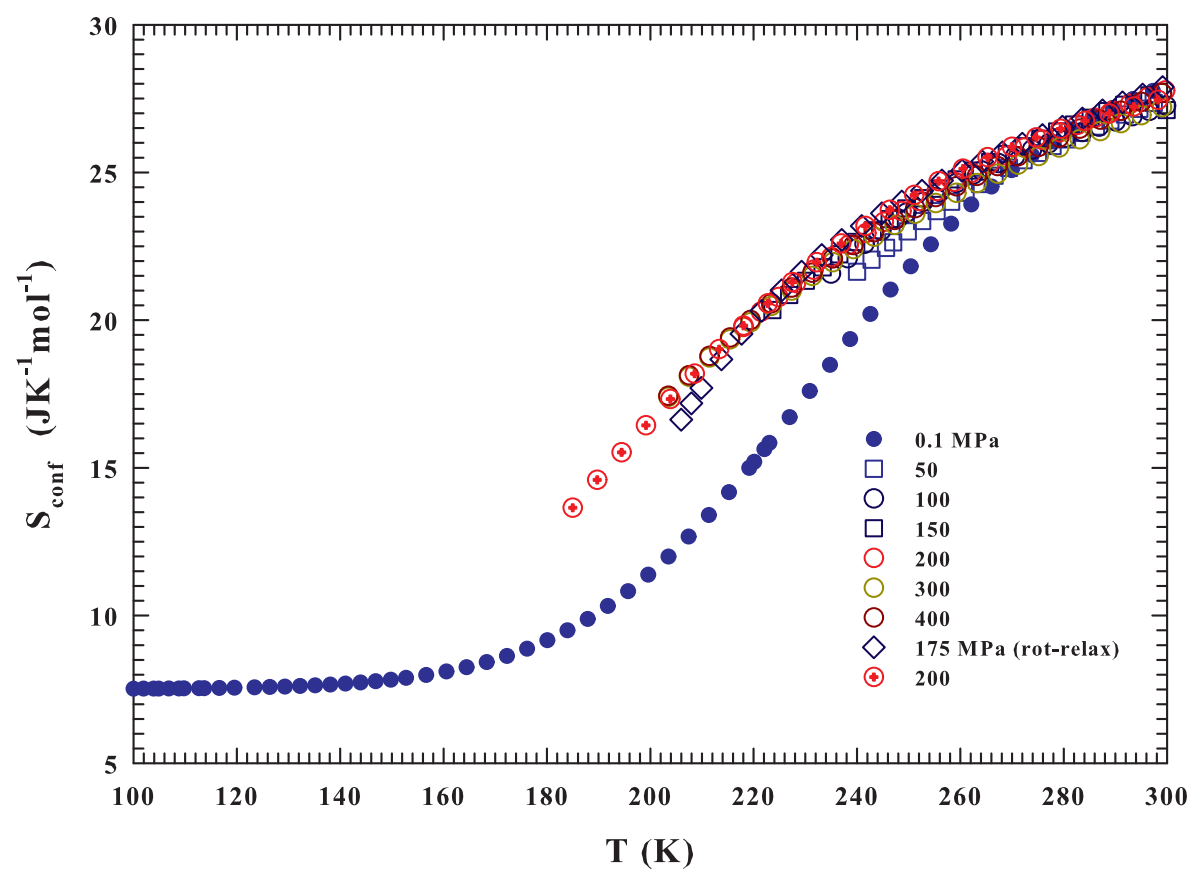

Figure 6. The $S_{\text {conf }}$ isobars calculated according to the AG theory are illustrated, at different pressures, in a linear scale for the temperature range $100-300 \mathrm{~K}$.

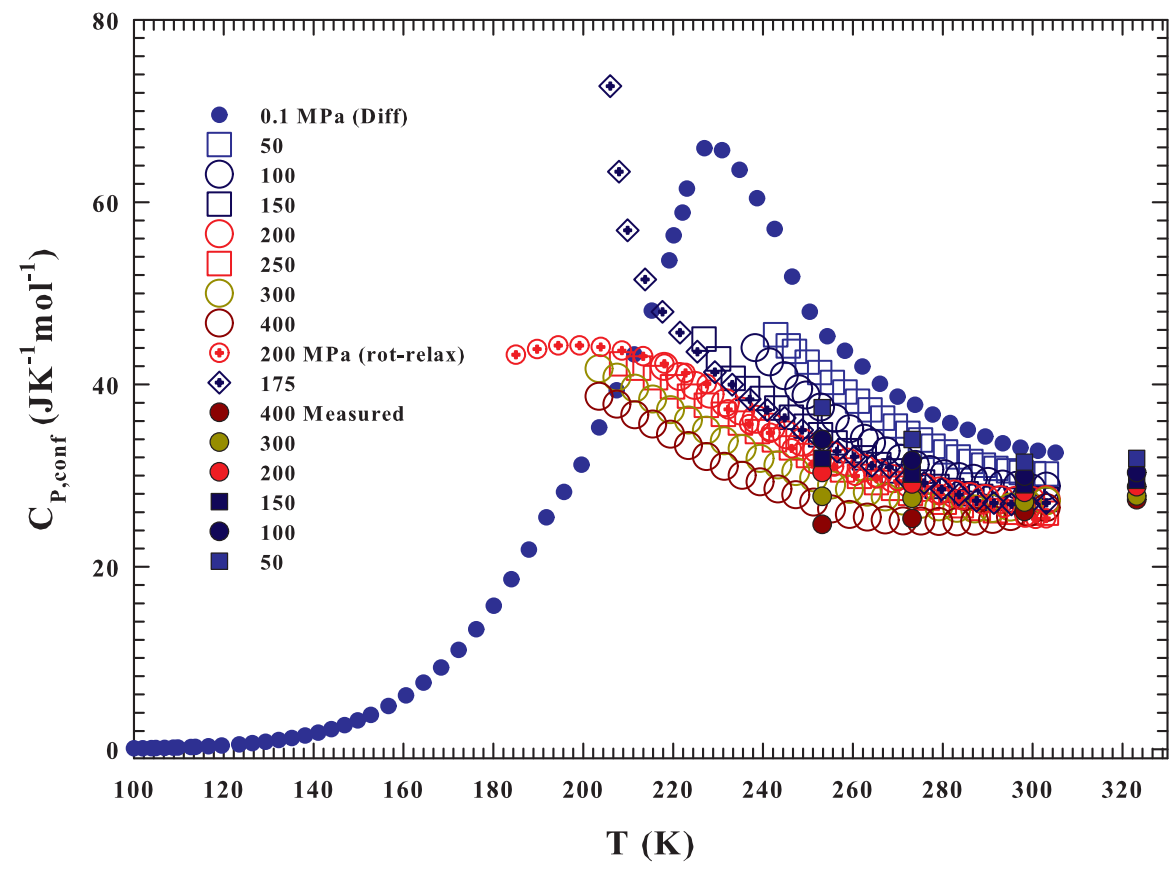

Figure 7. The $C_{P, \text { conf }}$ isobars calculated according to the AG procedure by using the bulk water transport data, in the $P$ range $0.1-400 \mathrm{MPa}$, are shown. For comparison, the bulk water experimentally measured $\Delta C_{P}$ at 50,100, 150, 200, 300 and $400 \mathrm{MPa}$, are also reported [53].

All the $C_{P, \text { conf }}$ isobars calculated with this procedure are shown in Figure 7 , where are also reported, for comparison, the bulk water's experimentally measured $\Delta C_{P}$ at 50 , 100, 150, 200, 300 and $400 \mathrm{MPa}$ [53]. A reasonable agreement between the temperature behavior of these latter experimental data and those evaluated in accordance with the procedure used can be observed, and the symbol size represents the experimental error. The experimental values at 323 and $298 \mathrm{~K}$ were considered as reference values after the entropy 
derivative. The entire behavior of these data is representative of the thermodynamics of water liquids in this phase of metastability. In spite of the limited temperature region inside the supercooled region (these calorimetric data coming from bulk water), the thermal evolution of the corresponding different isobars fully supports the presence of a LLCP, also giving information on where it is located in the phase diagram. In fact, if we look only at the $C_{P, \text { conf }}$ data derived from self-diffusion a different growth rate in isobar values can be easily observed between the isobars between 0.1 and $150 \mathrm{MPa}$ and those for $P>200 \mathrm{MPa}$. The former data, at 50,100,150 MPa, are characterized by a divergent behavior that involves all data at all temperatures explored, even if they go more and more into the metastability regime (also if the lowest temperature value of the isobar at $150 \mathrm{MPa}$ is slightly lower than the $C_{P, \text { conf }}$ maximum of that at $0.1 \mathrm{MPa}$ ). In the opposite case, $P>200 \mathrm{MPa}$, not only is the configurational specific heat growth rate less pronounced but also accompanied by a moderate change in its slope and an inflection point. This latter point therefore suggests that the LLCP is located in a pressure between 150 and $200 \mathrm{MPa}$.

The overall situation becomes clearer if we consider the specific heat data, at the 175 and $200 \mathrm{MPa}$ isobars, coming from the rotational relaxation time $\tau_{\theta}$. In this case, the increase in the temperature interval within the metastable region highlights both a maximum, and thus a WL point in $C_{P, \text { conf }}$ at $200 \mathrm{MPa}(\sim 195 \mathrm{~K})$ and a very pronounced divergent behavior at $175 \mathrm{MPa}$. In addition, the maximum value in $C_{P, \text { conf }}$ at $200 \mathrm{MPa}\left(\simeq 44.3 \mathrm{JK}^{-1} \mathrm{~mol}^{-1}\right)$ is smaller than that measured at $0.1 \mathrm{MPa}\left(\simeq 66.1 \mathrm{JK}^{-1} \mathrm{~mol}^{-1}\right)$, whereas the highest value obtained for the $175 \mathrm{MPa}$ isobar is $\simeq 72.7 \mathrm{JK}^{-1} \mathrm{~mol}^{-1}$ at $206 \mathrm{~K}$. These further data, in agreement with the precedents due to self-diffusion, not only give confirmation of the presence of a LLT but also indicate the presence of LLCP located in the following region of the phase diagram: an isobar $P_{C}$ between 175 and $200 \mathrm{MPa}$ and a critical temperature $195<T_{C}<206 \mathrm{~K}$. Such a result obtained from dynamical data inside the no man's land, also by considering the full agreement with the finding coming from the isothermal compressibility (evaluated from the density data) studies [35,44,69], undoubtedly provide crucial information regarding our understanding of the strange behavior of liquid water. We stress that this resulting and complex evolution of the configurational modes of liquid water clearly shown both by the $S_{\text {conf }}$ and $C_{P, \text { conf }}$ isobars due to its polymorphism is certainly the result of its underlying energy configuration: the energy landscape or inherent basins of energy [65].

\section{Conclusions}

Starting from the idea that liquid water is dominated by a polymorphism generated by the HB interactions and that this polymorphism, made from the LDL and HDL, dominates the behavior of thermodynamical functions, we have analyzed a lot of experimental data that enter well inside the no man's land in order to determine what they can tell us about the presence of the LLT and more generally what they reveal about the LLCP hypothesis. By considering literature dynamical data obtained by means of the NMR technique, such as the self-diffusion coefficient $\left(D_{S}\right)$ and the spin-lattice $\left(T_{1}\right)$ relaxation times, measured, respectively, in bulk and emulsion water at several different isobars in the range 0.1 to $400 \mathrm{MPa}$, we found that their temperature dependence provides further evidence for the LLCP hypothesis. The spin-lattice times were analyzed, as the proton relaxation rate $\left(R_{1}=1 / T_{1}\right)$, according to the NMR current theoretical models [60-62] evaluating a reorientational correlation time $\left(\tau_{\theta}\right)$ of the order, in the stable liquid phase, of some picoseconds. Both these data evolve with $T$ by changes of several orders of magnitude, whereas their pressure dependence, almost nothing in the stable liquid phase $\left(T>T_{m}\right)$ and as an effect of the LDL HB networking of the LDL liquid, becomes more and more pronounced at lower temperatures.

Both these quantities, diffusion isobars first and then some of $\tau_{\theta}$, have been studied according to the Adam-Gibbs model, typical of glass-forming liquids. A situation was recently studied using the ambient pressure isobar, also considering data of confined water and the molten amorphous phase, and covering a very wide temperature range 
from the boiling temperature to that of LDA amorphous phase. The aim was to highlight the behavior of the water configurational entropy and the corresponding specific heat contribution in order to clarify the LLT behavior on approaching LLCP.

A comparison of the thermal behaviors of all the evaluated $C_{P, \text { conf }}$ isobars, in particular, the observed maxima and the evolutions of the diverging behaviors, clearly reveals the presence of the LLT and, with a reasonable approximation, the LLCP locus in the phase diagram. This latter situation is fully consistent with recently studies on the water thermodynamic functions obtained from experimental data $[35,44,69]$ and proper MD simulations [36-39,68]. Finally, we underline that the observed significant configurational evolutions due the liquid water polymorphism are related to the local potential minima, known as inherent structures (ISs), surrounded by potential energy basins [65].

Author Contributions: All authors contributed equally. All authors have read and agreed to the published version of the manuscript.

Funding: This research received no external funding.

Institutional Review Board Statement: Not applicable.

Informed Consent Statement: Not applicable.

Data Availability Statement: The data that support the findings of this study are available from the corresponding author upon reasonable request.

Acknowledgments: The DM work was supported by the European Project H2020 A-LEAF - 732840; LP and GP benefited from the national PRIN 2017 project (Italy).

Conflicts of Interest: The authors declare no conflict of interest.

\section{References}

1. Ball, P. Water as an active constituent in cell biology. Chem. Rev. 2008, 108, 74-108. [CrossRef]

2. Debenedetti, P.G.; Stanley, H.E. Supercooled and glassy water. Phys. Today 2003, 56, 40-46. [CrossRef]

3. Speedy, R.J.; Angell, C.A. Isothermal compressibility of supercooled water and evidence for a thermodynamic singularity at $-45{ }^{\circ}$ C. J. Chem. Phys. 1976, 65, 851-858. [CrossRef]

4. Poole, P.H.; Sciortino, F.; Essmann, U.; Stanley, H.E. Phase-behavior of metastable water. Nature 1992, 360, 324-328. [CrossRef]

5. Mallamace, F. The liquid water polymorphism. Proc. Natl. Acad. Sci. USA 2009, 106, 15097-15098. [CrossRef] [PubMed]

6. Mallamace, F.; Broccio, M.; Corsaro, C.; Faraone, A.; Majolino, D.; Venuti, V.; Liu, L.; Mou, C.Y.; Chen, S.-H. Evidence of the existence of the low-density liquid phase in supercooled, confined water. Proc. Natl. Acad. Sci. USA 2008, 104, 424-428. [CrossRef] [PubMed]

7. Xu, L.M.; Mallamace, F.; Yan, Z.Y.; Starr, F.W.; Buldyrev, S.V.; Stanley, H.E. Appearance of a fractional Stokes-Einstein relation in water and a structural interpretation of its onset. Nat. Phys. 2009, 5, 565. [CrossRef]

8. Lobban, C.; Finney, J.L.; Kuhs, W.F. The structure of a new phase of ice. Nature 1998, 391, 268-270. [CrossRef]

9. Mishima, O.; Calvert, L.D.; Whalley, E. Melting Ice-I at $77 \mathrm{~K}$ and $10 \mathrm{~K}$ bar-a mew method of making amorphous solid. Nature 1984, 310, 393-397. [CrossRef]

10. Mishima, O.; Calvert, L.D.; Whalley, E. An apparently first-order transition between two amorphous phases of ice induced by pressure. Nature 1985, 314, 76-78. [CrossRef]

11. Mishima, O. Relationship between melting and amorphization of ice. Nature 1996, 384, 546-550. [CrossRef]

12. Rapoport, E. Model for melting-curve at high pressure. J. Chem. Phys. 1967, 46, 2891. [CrossRef]

13. Nemethy, G.; Scheraga, H. Structure of Water and Hydrophobic Bonding in Proteins. I. A Model for the Thermodynamic Properties of Liquid Water. J. Chem. Phys. 1962, 36, 3382. [CrossRef]

14. Davis, C.M.; Litovitz, T.A. Two-State Theory of the Structure of Water. J. Chem. Phys. 1965, 42, 2563. [CrossRef]

15. Jhon, M.S.; Grosh, J.; Ree, T.; Eyring, H. Significant-Structure Theory Applied to Water and Heavy Water. J. Chem. Phys. 1966, 44, 1465. [CrossRef]

16. Kamb, B. Structure of high-pressure forms of ice. Science 1965, 150, 205. [CrossRef]

17. Burton, E.F.; Oliver, W.F. X-ray diffraction patterns of ice. Nature 1935, 135, 505-506. [CrossRef]

18. Loerting, T.; Salzmannm, C.; Kohl, I.; Mayer, E.; Hallbrucker, A. A second distinct structural "state" of high-density amorphous ice at $77 \mathrm{~K}$ and 1 bar. Phys. Chem. Chem. Phys. 2001, 3, 5355. [CrossRef]

19. Mishima, $\mathrm{O}$. Reversible first-order transition between two $\mathrm{H}_{2} \mathrm{O}$ amorphs at $\sim 0.2 \mathrm{GPa}$ and $\sim 135 \mathrm{~K}$. J. Chem. Phys. 1994, 100, 5910-5912. [CrossRef] 
20. Prielmeier, F.X.; Lang, E.W.; Speedy, R.J.; Lüdemann, H.-D. The pressure-dependence of self-diffusion in supercooled light and heavy-water. Ber. Bunsenges Phys. Chem. 1988, 92, 1111-1117. [CrossRef]

21. Cerveny, S.; Mallamace, F.; Swenson, J.; Vogel, M.; Xu, L.M. Confined water as model of supercooled water. Chem. Rev. 2016, 116, 7608-7625. [CrossRef] [PubMed]

22. Lang, E.; Lüdemann, H.D. Pressure and temperature-dependence of longitudinal proton relaxation-times in supercooled water to $-87^{\circ} \mathrm{C}$ and 2500 bar. J. Chem. Phys. 1977, 67, 718. [CrossRef]

23. Suzuki, Y.; Mishima, O. Experimentally proven liquid-liquid critical point of dilute glycerol-water solution at $150 \mathrm{~K}$. J. Chem. Phys. 2014, 141, 094505. [CrossRef] [PubMed]

24. Murata, K.-I.; Tanaka, H. Liquid-liquid transition without macroscopic phase separation in a water-glycerol mixture. Nat. Mater. 2012, 11, 436-443. [CrossRef] [PubMed]

25. Mallamace, F.; Baglioni, P.; Corsaro, C.; Spooren, J.; Stanley, H.E.; Chen, S.-H. Transport properties of supercooled confined water. Riv. Nuovo Cimento 2011, 34, 253. [CrossRef]

26. Xu, Y.; Petrik, N.G.; Scott Smith, R.; Kay, B.D.; Kimmel, G.A. Growth rate of crystalline ice and the diffusivity of supercooled water from 126 to 262 K. Proc. Natl. Acad. Sci. USA 2016, 113, 14921-14925. [CrossRef]

27. Sjöström, J.; Swenson, J.; Bergman, R.; Kittaka, S. Investigating hydration dependence of dynamics of confined water: Monolayer, hydration water and Maxwell-Wagner processes. J. Chem. Phys. 2008, 128, 154503. [CrossRef]

28. Mallamace, F.; Corsaro, C.; Stanley, H.E. A singular thermodynamically consistent temperature at the origin of the anomalous behavior of liquid water. Sci. Rep. 2012, 2, 993. [CrossRef]

29. Xu, L.M.; Kumar, P.; Buldyrev, S.V.; Chen, S.-H.; Poole, P.H.; Sciortino, F.; Stanley, H.E. Relation between the Widom line and the dynamic crossover in systems with a liquid-liquid phase transition. Proc. Natl. Acad. Sci. USA 2005, 102, 16558-16562. [CrossRef]

30. Ito, K.; Moynihan, C.T.; Angell, C.A. Thermodynamic determination of fragility in liquids and a fragile-to-strong liquid transition in water. Nature 1999, 398, 492. [CrossRef]

31. Chen, S.-H.; Mallamace, F.; Mou, C.Y.; Broccio, M.; Corsaro, C.; Faraone, A.; Liu, L. The violation of the Stokes-Einstein relation in supercooled water. Proc. Natl. Acad. Sci. USA 2006, 103, 12974-12978. [CrossRef] [PubMed]

32. Mallamace, F.; Corsaro, C.; Stanley, H.E. Possible relation of water structural relaxation to water anomalies. Proc. Natl. Acad. Sci. USA 2013, 110, 4899-4904. [CrossRef]

33. Kim, K.H.; Späh, A.; Pathak, H.; Perakis, F.; Mariedahl, D.; Amann-Winkel, K.; Sellberg, J.A.; Lee, J.H.; Kim, S.; Park, J.; et al. Maxima in the thermodynamic response and correlation functions of deeply supercooled water. Science 2017, 358, 1589-1593. [CrossRef] [PubMed]

34. Liu, L.; Chen, S.-H.; Faraone, A.; Yen, C.W.; Mou, C.Y. Pressure dependence of fragile-to-strong transition and a possible second critical point in supercooled confined water. Phys. Rev. Lett. 2005, 95, 117802. [CrossRef] [PubMed]

35. Mallamace, F.; Corsaro, C.; Mallamace, D.; Fazio, E.; Chen, S.-H. Some considerations on the water polymorphism and the liquid-liquid transition by the density behavior in the liquid phase. J. Chem. Phys. 2019, 151, 044504. [CrossRef]

36. Abascal, J.L.; Vega, C. Widom line and the liquid-liquid critical point for the TIP4P/2005 water model. J. Chem. Phys. 2010, 133, 234502. [CrossRef] [PubMed]

37. Abascal, J.L.; Vega, C. Note: Equation of state and compressibility of supercooled water: Simulations and experiment. J. Chem. Phys. 2011, 134, 186101. [CrossRef]

38. Ni, Y.; Skinner, J.L. Evidence for a liquid-liquid critical point in supercooled water within the E3B3 model and a possible interpretation of the kink in the homogeneous nucleation line. J. Chem. Phys. 2016, 144, 214501. [CrossRef]

39. Sellberg, J.A.; Huang, C.; McQueen, T.A.; Loh, N.D.; Laksmono, H.; Schlesinger, D.; Sierra, R.G.; Nordlund, D.; Hampton, C.Y.; Starodub, D.; et al. Ultrafast X-ray probing of water structure below the homogeneous ice nucleation temperature. Nature 2014, 510, 381-384. [CrossRef]

40. Mallamace, F.; Branca, C.; Broccio, M.; Corsaro, C.; Mou C.-Y.; Chen, S.-H. The anomalous behavior of the density of water in the range 30 K-373 K. Proc. Natl. Acad. Sci. USA 2007, 104, 18387-18391. [CrossRef]

41. Erko, M.; Wallacher, D.; Hoell, A.; Hauß, T.; Zizak, I.; Paris, O. Density minimum of confined water at low temperatures: A combined study by small-angle scattering of X-rays and neutrons. Phys. Chem. Chem. Phys. 2012, 14, 3852-3858. [CrossRef]

42. Bridgman, P.W. Water, in the liquid and five solid forms, under pressure. Proc. Am. Acad. Art. Sci. 1912, 47, 441-558. [CrossRef]

43. Palmer, J.C.; Poole, P.H.; Sciortino, F.; Debenedetti, P.G. Advances in Computational Studies of the Liquid-Liquid Transition in Water and Water-Like Models. Chem. Rev. 2018, 118, 9129-9151. [CrossRef] [PubMed]

44. Mallamace, D.; Corsaro, E.; Mallamace, F.; Stanley, H.E. Experimental tests for a liquid-liquid critical point in water. Sci. China-Phys. Mech. Astron. 2020, 63, 127001. [CrossRef]

45. Adam, G.; Gibbs, J.H. On temperature dependence of cooperative relaxation properties uìin glass forming liquids. J. Chem. Phys. 1965, 43, 139. [CrossRef]

46. Starr, F.; Angell, C.A.; Stanley, H.E. Prediction of entropy and dynamic properties of water below the homogeneous nucleation temperature. Phys. A 2003, 323, 51. [CrossRef]

47. Mallamace, F.; Corsaro, C.; Mallamace, D.; Fazio, E.; Chen, S.-H.; Cupane, A. Specific Heat and Transport Functions of Water. Int. J. Mol. Sci. 2020, 21, 622. [CrossRef]

48. Kumar, P.; Buldyrev, S.V.; Becker, S.R.; Poole, P.H.; Starr, F.W.; Stanley, H.E. Relation between the Widom line and the breakdown of the Stokes-Einstein relation in supercooled water. Proc. Natl. Acad. Sci. USA 2007, 104, 9575-9579. [CrossRef] 
49. Lang, E.W.; Girlich, D.; Lüdemann, H.D.; Piculell, L.; Müller, D. Proton spin-lattice relaxation rate in supercooled $\mathrm{H}_{2} \mathrm{O}$ and $\mathrm{H}_{2}{ }^{-17} \mathrm{O}$ under high pressure. J. Chem. Phys. 1990, 93, 4796. [CrossRef]

50. Handa, Y.P.; Mishima, O.; Whalley, E. High-density amorphous ice. III. Thermal properties. J. Chem. Phys. 1986, 84, 2766. [CrossRef]

51. Archer, D.G.; Carter, R.W. Thermodynamic properties of the $\mathrm{NaCl}+\mathrm{H}_{2} \mathrm{O}$ system. 4. Heat capacities of $\mathrm{H}_{2} \mathrm{O}$ and $\mathrm{NaCl}(\mathrm{aq})$ in cold-stable and supercooled states. J. Phys. Chem. B 2000, 104, 8563. [CrossRef]

52. Tombari, E.; Ferrari, C.; Salvetti, G. Heat capacity anomaly in a large sample of supercooled water. Chem. Phys. Lett. 1999, 300, 749. [CrossRef]

53. Lin, C.-W.; Trusler, J.P.M. The speed of sound and derived thermodynamic properties of pure water at temperatures between (253 and 473) $\mathrm{K}$ and at pressures up to $400 \mathrm{MPa}$. J. Chem. Phys. 2012, 136, 094511. [CrossRef] [PubMed]

54. Oguni, M.; Maruyama, S.; Wakabayashi, K.; Nagoe, A. Glass transitions of ordinary and heavy water within silica-gel nanopores. Chem. Asian J. 2007, 2, 514. [CrossRef] [PubMed]

55. Oguni, M.; Kanke, Y.; Namba, S. Thermal properties of the water confined within nanopores of silica MCM-41. AIP Conf. Proceed. 2008, 982, 34.

56. Stejskal, E.O.; Tanner, J.E. Spin diffusion measurements: Spin echoes in the presence of a time-dependent field gradient. J. Chem. Phys. 1965, 42, 288. [CrossRef]

57. Götze, W.; Sjögren, L. Relaxation processes in supercooled liquids. Rep. Prog. Phys. 1992, 55, 241. [CrossRef]

58. Mallamace, F.; Corsaro, C.; Mallamace, D.; Vasi, C.; Stanley, H.E. The thermodynamical response functions and the origin of the anomalous behavior of liquid water. Farad. Disc. 2013, 167, 95. [CrossRef] [PubMed]

59. Catalán, J.; del Valle, J.C. Molecule 1-Methyl-5-nitroindoline Probes the Structural Change of Liquid Water with Temperature. ACS Omega 2018, 3, 18930-18934. [CrossRef]

60. Abragam, A. The Principles of Nuclear Magnetism; Oxford. U. P.: London, UK, 1961.

61. Bloembergen, N.; Purcell, E.M.; Pound, R.V. Relaxation Effects in Nuclear Magnetic Resonance Absorption. Phys. Rev. 1948, 73, 679. [CrossRef]

62. Kubo, R.; Tomita, K. A general theory of magnetic resonace absorption. J. Phys. Soc. Jpn. 1954, 9, 888. [CrossRef]

63. Hindman, J.C.; Svirrnickas, A.; Wood, M. Relaxation processes in water-study of proton spin-lattice relaxation time. J. Chem. Phys. 1973, 59, 1517. [CrossRef]

64. Hindman, J.C. Relaxation processes in water-viscosity, self-diffusion, and spin-lattice relaxation-kinetic model. J. Chem. Phys. 1974, 60, 4488. [CrossRef]

65. Stillinger, F.H.; Weber, T.A. Hidden structure in liquids. Phys. Rev. A 1982, 25, 978. [CrossRef]

66. Mallamace, F.; Branca, C.; Corsaro, C.; Leone, N.; Spooren, J.; Chen, S.-H.; Stanley, H.E. Transport properties of glass-forming liquids suggest that dynamic crossover temperature is as important as the glass transition temperature. Proc. Natl. Acad. Sci. USA 2010, 107, 22457. [CrossRef] [PubMed]

67. Yip, S.; Short, M.P. Multiscale materials modelling at the mesoscale. Nat. Mat. 2013, 12, 774. [CrossRef] [PubMed]

68. Hestand, N.J.; Skinner, J.L. Perspective: Crossing the Widom line in no man's land: Experiments, simulations, and the location of the liquid-liquid critical point in supercooled water. J. Chem. Phys. 2018, 149, 140901. [CrossRef]

69. Mallamace, F.; Mensitieri, D.; Mallamace, D.; Salzano de Luna, M.S.; Chen, S.-H. Some aspects of the liquid water thermodynamic behavior: From the stable to the deep supercooled regime. Int. J. Mol. Sci. 2020, 21, 7269. [CrossRef] 